Volume 00, Number 0, Xxxx XXXX, Pages 000-000

$\mathrm{S}(\mathrm{XX}) 0000-0$

\title{
EXTREMAL METRICS ON GRAPHS I
}

\author{
DMITRY JAKOBSON AND IGOR RIVIN
}

\begin{abstract}
We define a number of natural (from geometric and combinatorial points of view) deformation spaces of valuations on finite graphs, and study functions over these deformation spaces. These functions include both direct metric invariants (girth, diameter), and spectral invariants (the determinant of the Laplace operator, or complexity; bottom non-zero eigenvalue of the Laplace operator). We show that almost all of these functions are, surprisingly, convex, and we characterize the valuations extremizing these invariants.
\end{abstract}

\section{INTRODUCTION}

There is a vast literature on the subject of extremal graph theory. There, the general approach is to consider a natural invariant (invariant with respect to isomorphism) of graphs, and to try to understand which graphs make the invariant as big as possible, subject to (presumably natural) constraints. Examples of such invariants are:

Girth - the length of the shortest cycle;

Diameter - the greatest distance between a pair of vertices;

Tree number - the number of spanning trees,

and some closely related spectral invariants: the "determinant of the Laplacian", the smallest positive eigenvalue of the Laplacian, and so on.

It is expected that graphs which are "good" with respect to any one of these invariant will be good with respect to the others (where by "good", we mean that the graph is either extremal, or close to it), and will have other (a priori unsuspected) nice combinatorial properties.

Extremal graph theory is a rather difficult subject, largely due to its intrinsically combinatorial nature (arguably it is this difficulty which attracts most of the practitioners).

A seemingly not very closely related subject is that of differential geometry. One of its central areas is that of "uniformization", or "optimal geometry". There, we are often given a fixed topological space, and we try

Received by the editors November 20, 2018.

Key words and phrases. graphs, extremal graph theory, deformation theory, uniformization.

I. Rivin would like to thank the École Polytechnique for its hospitality during the preparation of this paper. D. Jakobson was partially supported by the NSF.

(C)0000 (copyright holder) 
to find a metric on this space which maximizes some invariant. The actual invariants studied are very often similar to those mentioned above for graphs. The motivation, on the other hand, is sometimes the same as that of extremal graph theory, but sometimes there is an additional factor: it is hoped that the extremal metrics would give a canonical representation of the topological space, which renders its topological properties more transparent (for example, the study of the topology of the sphere would be much more difficult if we did not have its standard "round" representation at our disposal).

Our motivation stems from both the areas sketched above: we would like to get canonical representations of graphs, but we have other concerns as well. First of all, the space of edge valuations of a given finite graph is a much simpler space than the space of metrics on a given topological space. Thus, we hope that the answers to our questions will be technically simpler than the corresponding differential-geometric results, but that the model is sufficiently rich to suggest what one might expect. By the same token, the space of edge valuations on a fixed graph is a much simpler space than the (discrete) space of graphs, though the latter is naturally embedded in the former. We thus hope to get insight into problems in extremal graph theory as well.

0.1. Outline of the paper. We set up the basic deformation spaces and announce the main convexity results in Section 11. We set up the girth problem in Section 2, and characterize the extremal valuations in Section 1 .

We define the basic matrices and operators we are working with, and show the convexity of the bottom eigenvalue and the complexity in Section 3. We characterize valuations extremal for complexity (or "determinant of laplacian" in Section 5, and valuations extremal for the bottom eigenvalue in Section 6. Finally, in Section 7 we analyse completely those graphs which are extremal for $\lambda_{1}$, under the additional assumption (which turns out to be very strong), that $\lambda_{1}$ appears without multiplicity.

\section{The Foundations}

We will always consider a fixed finite simple graph $G$. We will consider the following deformation spaces of edge valuations on $G$ :

$$
P(G)=\left\{f: E(G) \rightarrow \mathbb{R}^{+} \quad\left|\sum_{e \in E(G)} f(e)=\right| E(G) \mid\right\},
$$

- the space of all edge valuations of $G$.

$$
T(G)=\left\{f \in P(G) \quad \mid \sum_{e_{i} \text { incident to } v}=d_{v}, \forall v \in V(G)\right\}
$$


$C(G)=\left\{f \in P(G) \mid \exists g: V(G) \rightarrow \mathbb{R}^{+}\right.$, such that $\left.f\left(e_{v w}\right)=g(v)+g(w)\right\}$

The letters $T$ and $C$ are meant to suggest Teichmüller space and conformal deformation space respectively.

All three spaces have a natural linear structure, which we will use without further comment.

We will look at the variation of following invariants (defined below) over the above-described deformation spaces: girth $g$, bottom positive eigenvalue $\lambda_{1}$ of the Laplacian $\Delta$ and $\log \operatorname{det}^{*} \Delta$.

The first striking observation about these invariants is the following:

Theorem 1. The quantities $-g,-\lambda_{1},-\log \operatorname{det}^{*} \Delta$ are convex on $P(G)$ (and hence on its linear subspaces $C(G)$ and $T(G)$ ).

(The proofs of these results are spread out through this paper: The convexity of girth is given in Section 2; the convexity of $-\log \operatorname{det}^{*} \Delta-$ by Theorem 4, and the convexity of $\lambda_{1}$ is outlined in Section 3.2.)

Remark 1. It can also be shown that the "topological entropy of the geodesic flow", defined in terms of a different deformation of the adjacency matrix, is also convex. This is done in the article [Riv99] by the second author.

The convexity has far-reaching consequences. To wit, for every invariant $I \in\left\{g, \lambda_{1},-\log \operatorname{det}^{*} \Delta\right\}$, and for each deformation space $D \in\{P, C, T\}$ there is a unique canonical edge valuation $G_{D}^{I}$ maximizing the invariant. A natural question is one of the characterization of these critical valuations, and of understanding the relationship between the various $G_{D}^{I}$ for the different choices of $I$ and $D$.

Some properties follow immediately from the convexity, in particular:

Observation 1. If $G$ possesses a group $\Gamma(G)$ of automorphisms, then the weights of the critical points $G_{D}^{I}$ are invariant by these symmetries, thus, if the automorphism group of $G$ is edge transitive, then $G_{D}^{I}$ are all equal (independently of invariant and deformation space), and are given by the constant weighing on the edges. If the automorphism group of $G$ acts vertextransitively, then $G_{C}^{I}$ is given by the constant weighing.

Remark 2. A large class of graphs the automorphism group of which is vertex-but not edge-transitive is given by the Cayley graphs of finite groups.

For graphs not known a priori to be symmetric, the supposition that the unweighted graph $G$ is critical for one of the invariants, implies strong symmetry properties. For example, if $G$ is maximal for $\log \operatorname{det}^{*} \Delta$, then there is the same number of spanning trees through every edge of $G$ ( $G$ is equiarboreal in the terminology of Godsil). If $G$ is the maximum for $\lambda_{1}$ then, with rare exceptions, $\lambda_{1}$ occurs with multiplicity in the spectrum of $\Delta(G)$. If $G$ is maximal for girth, then every edge of $G$ is contained in a shortest 
cycle (the precise somewhat stronger statement is the content of Theorem (7).

\section{GIRTH}

The "direct" (girth) and "spectral" invariants are somewhat different conceptually. First we remind the reader that the length of a path in a weighted graph $G$ is the sum of the weights of the edges in the path. The girth $\gamma(G)$ is the length of the shortest cycle in $G$. The distance between two vertices of $G$ is the length of the shortest path connecting them; the diameter $D(G)$ is equal to the largest such distance. Thus, if $\mathfrak{C}$ is the set of all cycles of $G$, then the girth is given by:

$$
g=\min _{C \in \mathfrak{C}} \sum_{e \in C} f(e),
$$

where $f(e)$ is the valuation of the edge $e$. Note that each of the terms $\sum_{e \in C} v(e)$ is a linear function of the valuation $f$, and hence we have the immediate

Theorem 2. The girth $g(f)$ is a concave function on $P(G)$.

Proof. This follows from the observation that the minimum of a collection of concave (in particular linear) functions is concave. We leave the proof as an exercise to the interested reader.

For two vertices $u, v$ of $G$ denote by $\Lambda(u, v)$ the set of all paths $\lambda$ in $G$ connecting $u$ and $v$ (we can assume without loss of generality that the paths are not self-intersecting, to make sure that the number of paths considered is finite). Thus,

$$
d(u, v)=\min _{\lambda \in \Lambda} \sum_{e \in \lambda} f(e) .
$$

The diameter of $G$ is thus given by:

$$
D(G)=\max _{u, v \in V(G)} d(u, v) .
$$

Note that the diameter is not a priori convex, due to the additional maximum, though some of the methods we use for girth can be brought to bear on the diameter question as well.

\section{SPeCtral InVARIANTS}

Let $G$ be a graph with $n$ vertices and $m$ edges (we denote the set of such graphs by $\mathcal{G}_{m, n}$ ), and $f: E(G) \rightarrow \mathbb{R}$ be a valuation on the edges of $G$ (in the sequel the valuations are always assumed positive, but this is not essential for the definitions below). The adjacency matrix $A(G)$ (or just $A$, when no ambiguity is possible) of a graph $G \in \mathcal{G}_{m, n}$ is a square matrix of size $n$ where $A_{i, j}$ is weight of the edge joining the vertices $v_{i}$ and $v_{j}$ if there such an edge, and 0 otherwise. We always consider loopless graphs, so $A_{i i}=0$. 
The nearest neighbor Laplacian $\Delta$ acts on functions on the set $V(G)$ of the vertices of $G$ : given $g: V(G) \rightarrow \mathbb{R}, \Delta(g)(v)=\sum_{w \sim v} f(v w)(g(v)-g(w))$. (We exceptionally use $v w$ to denote the edge joining $v$ to $w$ ). Let $\Delta=\left\{L_{i j}\right\}$ be the matrix of $\Delta(G)$ of a (not necessarily simple) graph $G$; then $L_{i i}$ is the degree of the vertex $v_{i}$, and $-L_{i j}$ is the number of the edges joining $v_{i}$ and $v_{j} \neq v_{i}$ (equal to 0 or 1 for simple graphs). For $k$-regular graphs $\Delta=k \mathrm{Id}-A$.

Let $A$ be the adjacency matrix of a weighted graph $G$ with $n$ vertices, let $\delta$ be the maximal degree of a vertex in $G$, and let its spectrum (in the decreasing order) be given by

$$
\delta \geq \mu_{1}>\mu_{2} \geq \mu_{3} \geq \ldots \geq \mu_{n} \geq(-\delta) .
$$

The spectrum of $\Delta(G)$ is $0=\lambda_{0}<\lambda_{1} \leq \ldots \leq \lambda_{n-1}$. For $k$-regular graphs, $\delta=k=\mu_{1}$ and $\lambda_{j}=k-\mu_{j+1}, j=0,1, \ldots, n-1$. Note that the 0 is always in the spectrum of $\Delta(G)$ independently of the weighing on the edges, and, furthermore, as long as the weighing is strictly positive, and the graph $G$ is connected, the eigenspace of 0 is spanned by the vector $\mathbf{1}=(1, \ldots, 1)$.

3.1. Complexity of a graph. An important invariant of an unweighed graph $G$ is the number $\tau(G)$ of spanning trees of $G$; it is sometimes called the complexity of $G$. By Kirckhoff's theorem ([Kir]),

$$
n \tau(G)=\lambda_{1} \lambda_{2} \cdot \ldots \cdot \lambda_{n-1}
$$

and $\tau(G)$ is equal to the determinant of any cofactor of the matrix $\Delta$ and (it is some times called the determinant of Laplacian).

The definitions for weighed graphs are the essentially the same, except that

$$
\tau(G)_{f}=\sum_{T \in \text { spanning trees of } G e \in E(T)} \prod_{e(e) .}
$$

This has a natural interpretation in the framework of electrical circuits, where $f(e)$ is thought of as the conductance of the edge $e$. See Bol98.

3.2. Variational problems. The functions we consider are: The bottom nonzero eigenvalue $\lambda_{1}$ and

$$
\log \operatorname{det} \Delta^{*}=\sum_{i=1}^{n-1} \log \lambda_{i} .
$$

The bottom non-trivial eigenvalue $\lambda_{1}$ can be alternatively defined by the Rayleigh-Ritz quotient:

$$
\lambda_{1}=\min _{\sum_{i=1}^{n} x_{i}=0} \frac{\langle x, \Delta x\rangle}{\langle x, x\rangle}
$$

From this definition, the concavity of $\lambda_{1}$ over $P(G)$ is immediate. The concavity of $\log \operatorname{det} \Delta^{*}$ is somewhat trickier. First we show: 
Theorem 3. The logarithm of the determinant is a concave function on the set of positive definite symmetric matrices

Proof. Let $Q$ be such a matrix, and let

$$
Q(t)=Q+t B, \quad t \in \mathbb{R}
$$

be a line of symmetric matrices through $Q$. Then

$$
\frac{d \log \operatorname{det}(Q(t))}{d t}=\operatorname{tr}\left(B Q^{-1}\right)
$$

and

$$
\frac{d^{2} \log \operatorname{det}(Q(t))}{d t^{2}}=-\operatorname{tr}\left(B Q^{-1} B Q^{-1}\right)
$$

It suffices to show that the last trace is strictly positive. The matrix $R=Q^{-1}$ is positive definite, so can be conjugated by an orthogonal matrix $P$ to a diagonal matrix $D$, where $D_{i i}>0$. So, we can rewrite

$$
\operatorname{tr}(B R B R)=\operatorname{tr}\left(B O D O^{t} B O D O^{t}\right)=\operatorname{tr}\left(\left(O^{t} B O\right) D\left(O^{t} B O\right) D\right) .
$$

Let $B^{\prime}=O^{t} B O . \quad B^{\prime}$ is still symmetric. We see that $\operatorname{tr}(B R B R)=$ $\operatorname{tr}\left(B^{\prime} D B^{\prime} D\right)$. Now, let $d$ be the vector of the diagonal entries of $D$. It is not hard to check that $\operatorname{tr}\left(B^{\prime} D B^{\prime} D\right)=d^{t} \mathcal{B} d$, where $\mathcal{B}_{i j}=b_{i j}^{2}$. Note, however, that by our assumptions, all the entries of $d$ are strictly positive, so $d^{t} \mathcal{B} d>0$, and the result follows.

Now we can prove

Theorem 4. The function $\log \operatorname{det} \Delta^{*}$ is concave on $P(G)$.

Proof. The vector $\mathbf{1}=(1, \ldots, 1)$ is the zero eigenvector of $\Delta\left(G_{f}\right)$ for any edge-valuation $f$ in $P(G)$. Thus, the restriction $\Delta^{*}$ of $\Delta$ to the orthogonal complement of the subspace generated by $\mathbf{1}$ is a symmetric positive-definite operator, whose entries as a matrix, furthermore, are obviously linear in those of $\Delta$, no matter which basis of $\mathbf{1}^{\perp}$ we take. The result now follows immediately from Theorem 3

In the sequel, we characterize the extremal valuations for girth, $\lambda_{1}$, and $\log \operatorname{det} \Delta^{*}$ on our deformation spaces $P, C$, and $T$.

\section{Maximal girth valuations}

4.1. Maximum in $P(G)$. Let $G$ be a fixed graph, and suppose that $f_{\max } \in$ $P(G)$ is such that the $g(f)$ is maximal. There are two, somewhat different, cases to consider: the first is when $f_{\max }$ is an interior point of $P(G)$ (i.e. no $f(e)$ vanishes), the second is when $f$ is a boundary point (so that one for one or more edges $e, f(e)=0)$. We will examine the interior point case first, since it contains the crucial ideas, and is slightly simpler. 
4.2. Interior maximum. The idea is that we use something like a piecewiselinear version of Lagrange multipliers. To wit, suppose that $f_{\max }$ is our maximal point. That means that there is a collection of cycles $C_{1}, \ldots, C_{n}$, such that $\ell\left(C_{i}\right)=g_{f}$, while $\ell(C)>g_{f}$ for any other cycle $C$. Consider a small perturbation $g$ of the valuation $f: f_{1}=f+t h$. Since $g$ still has to lie in $P(G)$, we must have $\langle h, \mathbf{1}\rangle=0$. The condition that $f$ is maximal is equivalent to saying that $g_{f_{1}} \leq g_{f}$. However, for $t$ sufficiently small, a shortest cycle for the valuation $f_{1}$ has to be one of the cycles $C_{1}, \ldots, C_{n}$, thus the hypothesis that $g_{f_{1}} \leq g_{f}$ means that $\min \ell\left(C_{i}\right)$ at the weighing $f_{1}$ has to be smaller than $g_{f}$. Consider the quantities $H_{i}=\sum_{e \in C_{i}} h(e)$. We know that at least one of them has to be negative, but this (by multiplying by -1 if necessary) is so if and only if $\exists i, j$, such that $\operatorname{sgn} H_{i}=-\operatorname{sgn} H_{j}$, or else all the $H_{i}$ vanish. The necessary and sufficient conditions follow from Farkas' Lemma:

Theorem 5 (Farkas Lemma). Let $v_{1}, \ldots, v_{n}, u \in \mathbb{R}^{k}$. Then there exists a vector $w \in \mathbb{R}^{k}$, such that $\left\langle w, v_{i}\right\rangle \geq 0, \quad 1 \leq i \leq n$ (at least one inner product being positive) and $\langle u, w\rangle=0$ if and only if $u$ is not in the open convex cone generated by the $v_{i}$.

Remark. $u$ is in the convex cone generated by the $v_{1}, \ldots, v_{n}$ if there exist $\mu_{1}, \ldots, \mu_{n}$ either all negative or all positive, such that $u=\sum_{i=1}^{n} \mu_{i} v_{i}$.

Proof of Farkas Lemma. Suppose first that

$$
u=\sum_{i=1}^{n} \mu_{i} v_{i}, \quad \mu_{i}>0,1 \leq i \leq n .
$$

Take any $w$ such that $\langle w, u\rangle=0$. Then

$$
0=\langle w, u\rangle=\sum_{i=1}^{n} \mu_{i}\left\langle w, v_{i}\right\rangle .
$$

Since the $\mu_{i}$ are all positive, not all of the inner products $\left\langle w, v_{i}\right\rangle$ can be positive, so $w$ does not satisfy the hypotheses of the theorem.

Suppose now that $u$ is not in the open cone $C$ generated by $v_{1}, \ldots, v_{n}$. Consider the projection of $C$ onto the subspace $u^{\perp}$ orthogonal to $u$. This is again an open convex cone $C_{u}$, which omits at least one point of $u^{\perp}$ (the origin). Therefore it is a proper cone, and is thus contained in a half-space $H^{+}$, and thus the positive normal vector to $\partial H^{+}$has positive inner product with any vector in the projection of $C$, and hence with any vector in $C$ (since a vector in $C$ can be written as a sum of a vector in $C_{u}$ with a multiple of $u)$.

Theorem 5 can be generalized as follows:

Theorem 6. Let $v_{1}, \ldots, v_{n}, u_{1}, \ldots, u_{m} \in \mathbb{R}^{k}$. Then there exists a vector $w \in \mathbb{R}^{k}$, such that $\left\langle w, v_{i}\right\rangle \geq 0, \quad 1 \leq i \leq n$ (with at one inner product 
positive) and $\left\langle u_{j}, w\right\rangle=0, \quad 1 \leq j \leq m$ if and only if no linear combination $\sum_{j=1}^{m} a_{j} u_{j}$ is in the open convex cone generated by the $v_{i}$.

Proof. If some linear combination $u=\sum_{j=1}^{m} u_{j}$ lies in the open cone $C$, then the same argument as in the beginning of the proof of Theorem 5 shows the non-existence of the requisite $w$. Otherwise, if $u_{1}, \ldots, u_{j}$ span $\mathbb{R}^{k}$, there is nothing left to prove. Assume then that they span a proper subspace $U$, and project $C$ onto the orthogonal complement, to get $C_{U} \cdot C_{U}$ omits the origin by assumption, and the same argument as in the proof of Theorem 5 completes the proof.

Remark 3. The above theorems 5 and 6 do not address the question of when the there exists a nonzero vector such that the inner products with the $u_{j}$ and $v_{i}$ are all zero. This, however, is obviously true if and only if the span of all of the $v_{i}$ together with all of the $u_{j}$ is a proper subspace of $\mathbb{R}^{k}$.

Theorems 5 and 6 and Remark 3 combine to give the following characterization of the extremal points of girth in $P(G), T(G)$ and $C(G)$, which we state in the Theorem 0 below. First

Notation. The systoles of $G$ corresponding to a weighing $f$ are cycles $s_{1}, \ldots, s_{k}$ whose length is equal to the girth of $G$ with the weighing $f$. We call edge systoles the vectors $\mathfrak{s}_{1}, \ldots, \mathfrak{s}_{k}$ in $\mathbf{R}^{E(G)}$ whose $e$-th coordinate is 1 if $e$ is contained in the corresponding cycle $s_{j}$. We call the vertex systole corresponding to $s_{i}$, the vector $\sigma_{i}$ in $\mathbb{R}^{V(G)}$, whose $v$-th coordinate is 1 if $v$ is incident to $s_{i}$, and 0 otherwise. The vertex vector $w_{v}$ is the vector in $\mathbb{R}^{E(G)}$ whose $e$-th coordinate is 0 unless $e$ is incident to the vertex $v$, in which case the coordinate is 1 . The degree vector $d(G)$ is the vector in $\mathbb{R}^{V(G)}$ whose $v$-th coordinate is the degree of the vertex $v$.

Theorem 7. A weighting $f \in P(G)$ is maximal for girth if and only if the constant vector 1 lies in the open cone generated by the edge systoles of $G$ with the weighting $f$. The maximal weighing $f$ is unique if and only if the edge systoles of $G$ corresponding to the weighing $f$ together with the constant vector span the whole space $\mathbb{R}^{E(G)}$.

A weighing $f$ in $T(G)$ is maximal for girth if and only if some linear combination of the vertex vectors $w_{1}, \ldots, w_{V_{G}}$ lies in the open cone generated by the edge systoles of $G$ with the weighing $f$. The maximal weighing $f$ is unique if and only if the edge systoles and the vertex vectors span $\mathbb{R}^{E(G)}$.

A weighing $f$ in $C(G)$ is maximal for girth if and only if the degree vector $d(G)$ is contained in the open cone generated by the vertex systoles of $G$. The maximal weighing is unique if and only if the degree vector together with the vertex systoles span $\mathbb{R}^{V(G)}$. 


\section{THE TREe NUMBER}

By the weighted version of Kirckhoff's theorem ( Bol98])

$$
\tau(G)=\sum_{T \in \mathcal{T}(G)} \prod_{e_{j} \in T} x_{j}
$$

where the sum is taken over the set $\mathcal{T}(G)$ of the spanning trees of $G$.

We will find necessary and sufficient condition for a valuation $f$ to be the critical point for $\tau(G)$ (which is the same as being maximal by $\log \operatorname{det} \Delta^{*}$, by the discussion in the Introduction) on $P(G), C(G)$ and $T(G)$. It should be noted that such a critical point might not exist, and we might have to look for boundary maxima. Our methods can be easily adapted to deal with those cases as well, and since writing down the conditions is somewhat more cumbersome, we leave this to the reader.

5.1. Maximum in $P(G)$. We start with $P(G)$, since the result in that case is the simplest to state, and seems, at least at the moment to have the simplest combinatorial interpretation. Finding the maximum of $\tau(G)$ on $P$ is a Lagrange multiplier problem. The condition for $x \in P(G)$ to be a critical point for $\tau(G)$ is

$$
\frac{\partial \tau(G)}{\partial x_{1}}=\frac{\partial \tau(G)}{\partial x_{2}}=\ldots=\frac{\partial \tau(G)}{\partial x_{m}}
$$

The partial derivatives above are given by

$$
\tau_{j}=\frac{\partial \tau(G)}{\partial x_{j}}=\sum_{e_{j} \in T \in \mathcal{T}(G)} \prod_{k \neq j ; e_{k} \in T} x_{k} .
$$

The ratio $\tau_{j} / \tau(G)$ is called the effective resistance of $e_{j}$.

We have thus proved:

Proposition 1. The graph valuation $f$ is maximal for $\tau(G)$ in $P(G)$ if and only if the effective resistances of all edges are the same.

If an unweighted graph satisfies the assumptions of Proposition 1 then every edge of this graph is contained in the same number of spanning trees. Such graphs were studied by Godsil in God81; he calls these graphs equiarboreal. Obviously, all edge-transitive graphs (the automorphism group acts transitively on the edges) are equiarboreal. Godsil gives several more sufficient conditions for a graph to be equiarboreal; in particular, any distanceregular graph and any color class in an association scheme is equiarboreal (the least restrictive condition Godsil gives is for a graph to be 1homogeneous). By an easy counting argument one can show that for an unweighted equiarboreal graph

$$
T_{1}=T_{2}=\ldots=\tau(G) \cdot(n-1) / m
$$

\footnotetext{
${ }^{1}$ See [Bou] for examples of edge-transitive graphs which are not vertex-transitive.
} 
where $T_{j}$ is the number of spanning trees containing $e_{j}$ (this is actually the result of Foster, cf. Fos ) so the necessary condition for a graph to be equiarboreal is that $m$ divide $(n-1) \tau(G)$.

Remark 4. Any tree is equiarboreal.

We remark that the graphs which have the most spanning trees among the regular graphs with the same number of vertices are not necessarily equiarboreal, and vice versa. For example, the 8-vertex Möbius wheel (cf. [Big93]) which has the most spanning trees among the 8-vertex cubic graphs is not equiarboreal (cf. also Val]), while the cube (which is certainly edgetransitive, hence equiarboreal) has the second biggest number of spanning trees among the 8-vertex cubic graphs.

5.2. Maxima in $T(G)$ and $C(G)$. The Lagrange multiplier method of the previous section works just as well in $T(G)$ and $C(G)$. We leave the (easy) computation to the reader, and just summarize the results in

Theorem 8. A valuation $f$ is maximal in $T(G)$ if and only if there exists constants $\lambda_{1}, \ldots, \lambda_{V(G)}$, such that if the edge e has endpoints $v_{i}$ and $v_{j}$, then

$$
\tau(e)=\lambda_{i}+\lambda_{j}
$$

$A$ valuation $f$ is maximal in $C(G)$ if and only if for any two vertices $v$ and $w$

$$
\operatorname{deg} w \sum_{e \text { incident to } v} \tau(e)=\operatorname{deg} v \sum_{e \text { incident to } w} \tau(e) .
$$

If we ask the same question as previously - when is the constant valuation maximal? - the condition for a maximum in $T(G)$ does not appear to have an obvious combinatorial interpretation. The condition for the maximum in $C(G)$ can be restated in the following way:

Corollary 1. Let $d_{T}(v)=\sum_{\text {spanning trees } T} \operatorname{deg} v$ in $T$. Then, if the constant valuation is maximal for $\tau(G)$ on $C(G)$, then for any two vertices $v$ and $w$,

$$
\frac{d_{T}(v)}{\operatorname{deg} v}=\frac{d_{T}(w)}{\operatorname{deg} w}
$$

\section{Eigenvalues of the Laplacian}

To find the condition for maximality with respect to the bottom non-zero eigenvalue of the Laplacian, we will use the Rayleigh-Ritz characterization of of $\lambda_{1}$. This implies immediately that:

Theorem 9. Let $f$ be the weighing on $G$ (in our application, $S$ could be any one of $P(G), T(G), C(G)$, but it could be anything). Let $E_{\lambda_{1}}$ be the eigenspace corresponding to $\lambda_{1}$. Let $Q$ be any infinitesimal variation (that is, an element of the tangent space of $S$ ) of the valuation, and $Q_{\Delta}$ the induced variation of the Laplacian matrix. Then the quadratic form given 
by $Q_{\Delta}$ restricted to $E_{\lambda_{1}}$ is indefinite if and only if $f$ is maximal with respect to $\lambda_{1}$.

Proof. The argument is a version of that given in the beginning of section 4.2. We use the Rayleigh-Ritz quotient characterization (given in eq. 3). The space $E_{\lambda_{1}}$ is precisely the set of vectors where the minimum is attained, so at any unit vector $x \notin E_{\lambda_{1}},\left\langle x,\left(\Delta+t Q_{\Delta}\right) x\right\rangle$ is strictly greater than $\left\langle y,\left(\Delta+t Q_{\Delta}\right) y\right\rangle$ for $y$ a unit vector in $E_{\lambda_{1}}$, for $t$ sufficiently small. Thus, the first variation of $\lambda_{1}$ is given by the first variation of $\lambda_{1}$ restricted to $E_{\lambda_{1}}$, and that is given precisely by the restriction of the quadratic form given by $Q_{\Delta}$. Now, if that were definite, we would be able to increase $\lambda_{1}$ by applying either the variation $Q_{\Delta}$ or $-Q_{\Delta}$.

Note now that the space of all possible variations of the Laplacian induced by changes in the edge valuations has a natural linear structure (one can think of it as a subspace of the tangent space to symmetric matrices). Call that space $V_{\text {var }}$. If $M \in V_{\text {var }}$, then $x^{t} M x$ can be thought of as a scalar product of $M$ with a vector $P_{x}$, whose $i j$-th coordinate is given by $x_{i} x_{j}$ (this is just the outer product of $x$ with itself, the letter $P$ is used to point out that when $x$ is a unit vector, $P_{x}$ is just the projection on the subspace generated by $x$ ). Let $\mathcal{P}_{\lambda_{1}}=\left\{P_{x} \mid x \in E_{\lambda_{1}}\right\}$. If $S^{\perp}$ is the orthogonal complement to the tangent space of the deformation $S$, Theorem 6 (whose proof does not use the finiteness of the sets involved) gives us:

Theorem 10. A valuation $f$ is maximal in $S$ with respect to $\lambda_{1}$ if and only if the intersection of $S^{\perp}$ with the open cone generated by $\mathcal{P}_{\lambda_{1}}$ is nonempty.

What is the "open cone generated by $\mathcal{P}_{\lambda_{1}}$ " ? It is an easy exercise to show that this is precisely the set of positive self-adjoint operators on $E_{\lambda_{1}}$ (that is, operators for which $E_{\lambda_{1}}$ is an invariant subspace ; which are positive on that subspace, and zero elsewhere). so Theorem 10 can be restated as:

Theorem 11. A valuation $f$ is maximal in $S$ with respect to $\lambda_{1}$ if and only if $S^{\perp}$ contains a positive self-adjoint operator $\Lambda$ on $E_{\lambda_{1}}$.

Corollary 2. If $E_{\lambda_{1}}$ is one-dimensional, then $f$ is maximal if and only if $S^{\perp}$ is spanned by $P_{v}$, where $v$ is a unit eigenvector of $\lambda_{1}$.

All the above might sound somewhat abstract, so let us now specialize to the the deformation spaces we have in mind. First, consider $P(G)$. In this case, it is easy to check that

$$
\frac{\partial \Delta}{\partial f(e)}=Q_{e}
$$

where, if the endpoints of $e$ are $v_{i}$ and $v_{j}$, then $Q_{i i}=Q_{j j}=1 ; Q_{i j}=Q_{j i}=$ -1 , and all of the other entries are 0 . The general variation of $\Delta$ is given by $Q_{\alpha}=\sum_{e} \alpha_{e} Q_{e}$, and in order to stay in $P(G)$, we must have $\langle\alpha, \mathbf{1}\rangle=0$.

It can be seen that the variation space $S$ of the Laplacians is spanned by the vectors $Q_{e_{0}}-Q_{e}$, where $e_{0}$ is an arbitrary fixed edge. 
6.1. The first eigenvalue $\lambda_{1}$ appears without multiplicity. If the eigenspace of $\lambda_{1}$ is one-dimensional, and the eigenvector is $v$, then by Corollary 2, $v^{t}\left(Q_{e_{0}}-Q_{e}\right) v=0$, for all $e$. If $e$ is an edge with endpoints $x$ and $y$, then a calculation shows that $v^{t} Q_{e} v=\left(v_{x}-v_{y}\right)^{2}$, and so for a maximal valuation, we must have

$$
v_{x}-v_{y}= \pm c
$$

for any adjacent pair of vertices $x, y$. We study graphs which have an eigenvector satisfying the condition given by eq. (7) in section 7 , but it is a priori clear that this condition is very rarely satisfied, and "usually" graphs maximal for $\lambda_{1}$ have a higher-dimensional first eigenspace. Curiously, the same holds for the (smaller) deformation spaces $T(G)$ and $C(G)$. Indeed, consider first $T(G)$. There, the deformation space of the Laplacians is spanned by matrices $Q_{e_{1}}-Q_{e_{2}}$, where $e_{1}$ and $e_{2}$ have a vertex in common. Thus, the same computation as that leading to eq. (17) gives that the eigenvector of $\lambda_{1}$ for a critical graph must satisfy:

$$
v_{x}-v_{y}= \pm c_{x}
$$

for any adjacent pair of vertices $x, y$. A priori, this seems somewhat weaker than the condition (ब) (since $c_{x}$ now depends on $x$ ), but in fact it is clear that for a connected graph $G$, it is equivalent ; the case of $G$ disconnected is different, but not particularly interesting.

For $C(G)$, the deformation space of Laplacians is generated by the differences $\operatorname{deg} w M_{v}-\operatorname{deg} v M_{w}$, where $M_{i}$ is the matrix whose $i i$-th entry is the degree of the $i$-th vertex; $M_{i j}$ is equal to -1 if $v_{j}$ is incident to $v_{i}$, likewise $M_{j i}$, and all other $M_{j k}$ are equal to 0 . If $v$ is a vector, then

$$
v^{t} M_{x} v=\operatorname{deg} x v_{x}^{2}-2 \sum_{y \sim x} v_{x} v_{y}=2 v_{x} \sum_{y \sim x}\left(v_{x}-v_{y}\right)-\operatorname{deg} x v_{x}^{2} .
$$

If $v$ is an eigenvector of $G$ with eigenvalue $\lambda$, then $\sum_{y \sim x}\left(v_{x}-v_{y}\right)=\lambda v_{x}$, and so

$$
v^{t} M_{x} v=(2 \lambda-\operatorname{deg} x) v_{x}^{2} .
$$

From the equation $v^{t}\left(\operatorname{deg} w M_{v}-\operatorname{deg} v M_{w}\right) v=0$, it follows that:

$$
\left(\frac{2 \lambda}{\operatorname{deg} x}-1\right) v_{x}^{2}=\left(\frac{2 \lambda}{\operatorname{deg} y}-1\right) v_{y}^{2} .
$$

In particular, note that when the graph $G$ is regular, it follows that

$$
\left|v_{x}\right|=\left|v_{y}\right| \text {, }
$$

for any two vertices $x, y$.

Remark. It is not difficult to construct regular graphs which have an eigenvector satisfying eq. (10): any such graph is constructed by taking an $l$ regular bipartite graph, whose vertex set is the union of the sets $R$ of red vertices and $B$ of black vertices, and constructing $k$-regular graphs with vertex sets $R$ and $B$ respectively (then adjoining their edge sets to that of 
the original bipartite graph). Then the function which is 1 on $R$ and -1 on $B$ is an eigenvector with eigenvalue $2 l$. It is much less clear that this can be done in such a way that $2 l$ is the lowest eigenvalue.

6.2. The general case. When the eigenspace of $\lambda_{1}$ has dimension possibly greater than 1, Theorem 11, compact with the finite-dimensional spectral theorem (that a positive self-adjoint operator can be diagonalized, with respect to an orthonormal basis, with positive weights) gives us the following extensions of the results of the previous subsection:

Theorem 12. In order for a valuation $f$ to be maximal for $\lambda_{1}$ with respect to $P(G)$, it is necessary and sufficient for there to be an orthogonal basis $v_{1}, \ldots, v_{d}$ of $E_{\lambda_{1}}$, and a collection of non-negative constants $c_{1}, \ldots, c_{d}$, not all zero, and a constant $c>0$ such that for any pair of adjacent vertices $x, y$ of $G$

$$
\sum_{i=1}^{d} c_{i}\left(v_{i}(x)-v_{i}(y)\right)^{2}=c .
$$

In order for $f$ to be maximal for $\lambda_{1}$ with respect to $T(G)$, the same condition (11) holds, assuming that $G$ is connected.

In order for $f$ to be maximal for $\lambda_{1}$ with respect to $C(G)$, there must be constants as above, such that for any vertex $x$ of $G$,

$$
\left(\frac{2 \lambda}{\operatorname{deg} x}-1\right) \sum_{i=1}^{d}\left(c_{i} v_{i}^{2}(x)\right)=c .
$$

Remark 5. The condition (11) gives an embedding of the edge set $E(G)$ into a $(d-1)$-dimensional ellipsoid

$$
\sum_{i=1}^{d} c_{i} z_{i}^{2}=c
$$

by the "differentials" $z_{i}=d v_{i}(e)=v_{i}(x)-v_{i}(y)$, where we have chosen an arbitrary orientation of the edge $e=(x y)$. The corresponding vertex condition gives, for a regular graph, a similar embedding of the vertex set $V(G)$ by the eigenvectors $v_{i}$.

\section{Graphs With an EIGENVECTOR OF CONSTANT GRADIENT}

We now study connected graphs which admit an eigenvector $f: V \rightarrow \mathbb{R}$ satisfying (7) for some $c \geq 0$. If $c=0$ then $f$ is a multiple of a constant vector and so has eigenvalue zero which is a contradiction. If $c \neq 0$ then it is easy to see that the graph $G$ cannot have odd cycles and hence is bipartite. Namely, let $u_{1} u_{2} \ldots u_{l}$ be a cycle. Then (putting $\left.u_{l}=u_{0}\right) \sum_{i=1}^{l}\left(f\left(u_{i}\right)-f\left(u_{i-1}\right)\right)=0$. But each term in the sum is equal to $\pm c$, and since the number of terms in the sum is odd, they cannot add up to 0 . 
We now want to study the unweighted $k$-regular graphs which have an eigenvector (corresponding to an eigenvalue $\mu>0$ ) satisfying (7) (without necessarily assuming that $\mu$ is simple). We shall rescale the eigenvector so that $c=1$ in (7). From (7) it follows that for each vertex $u$ the expression $\mu \cdot f(u)$ can only take one of the values $k, k-2, k-4, \ldots,-k+2,-k$. Consider first the vertex $u_{0}$ where $f(u)$ takes its maximal value $a$ (by changing the sign if necessary we can assume that $a>0$ ). It follows that $f$ takes value $a-1$ on all the neighbors of $u_{0}$, hence

$$
a \mu=k
$$

Next, consider any neighbor $u_{1}$ of $u$. The value of $f$ at any neighbor of $u_{1}$ can be either $a$ (let there be $r_{1} \geq 1$ such neighbors; $u_{0}$ is one of them); or $a-2$ (it follows that there are $k-r_{1}$ such neighbors). From the definition of the Laplacian it follows that

$$
\mu(a-1)=k-2 r_{1}
$$

It follows from the last two formulas that

$$
\mu=2 r_{1}
$$

where $r_{1} \geq 1$ is a positive integer. If $r_{1}=k$, then $\mu=2 k$ is the largest eigenvalue of $\Delta$.

We next define the level of a vertex $u$ to be equal to $j$ if $f(u)=a-j$; we denote the set of all vertices of $G$ at level $j$ by $G_{j}$. It is easy to see that if $u \in G_{j}$ has $r_{j}$ neighbors where $f$ takes value $a-j+1$ then

$$
\mu(a-j)=k-2 r_{j}
$$

It follows that $r_{j}$ is the same for all $u \in G_{j}$. Using (13) we see that

$$
r_{1} \cdot j=r_{j}
$$

Consider now a "local minimum" $u \in G_{N}$. Then $r_{N}=k$, and we see that

$$
r_{1} \mid k
$$

Let $n_{j}$ denote the number of vertices in $G_{j}$. Counting the vertices connecting $G_{j}$ and $G_{j+1}$ in two different ways, we see that for all $0 \leq j \leq N-1$,

$$
n_{j}\left(k-r_{j}\right)=n_{j+1} r_{j+1}
$$

Consider the case $r_{1}=1, \mu=2$. It follows from the previous calculations that $r_{j}=j$ and that $N=k$. Accordingly, $n_{j}=n_{0}\left(\begin{array}{l}k \\ j\end{array}\right)$ and

$$
|G|=2^{k} n_{0}
$$

We next describe a class of graphs admitting an eigenvector of $\Delta$ with $\mu=2$ satisfying (]).

An obvious example of such a $k$-regular graph is the $k$-cube, and any such graph has the same number of vertices as a disjoint union of $n_{0}$ cubes by (15). Start now with such a union, choose the partition of the vertices of each cube into "levels" and take two edges $u_{1} u_{2}$ and $u_{3} u_{4}$ in two different 
cubes such that $u_{1}, u_{3}$ are both in level $j$ while $u_{2}, u_{4}$ are both in level $j+1$. If we perform an edge switch

$$
\left(u_{1} u_{2}\right),\left(u_{3} u_{4}\right) \rightarrow\left(u_{1} u_{4}\right),\left(u_{3} u_{2}\right)
$$

then the number of the connected components of our graph will decrease while the eigenvector $f$ will remain an eigenvector with the same eigenvalue.

Performing sequences of edge switches as described above, we obtain examples of connected graphs satisfying (7) and (15) for any $n_{0}$. Conversely, it is easy to show that starting from a graph satisfying (7) and (15) and having chosen a partition of its vertices into levels one can obtain $n_{0}$ disjoint $k$-cubes by performing a sequence of edge switches as above.

We now want to consider the case when $\mu=\mu_{1}$ is the lowest eigenvalue of the Laplacian. The first remark is that then necessarily $\mu \leq k$, and $\mu=k$ only if $G=K_{k, k}$. Next, we want to consider "small" $k$ for which $k-2 \sqrt{k-1}$ (the "Ramanujan bound") is less than 2 (this happens for $3 \leq k \leq 6$ ). It then follows from the results of Alon ( $\mathbb{N i 1}$ ) that the diameter of $G$ (and hence the number of vertices in $G$ ) is bounded above.

Proposition 2. For $3 \leq k \leq 6$ there are finitely many $k$-regular graphs for which the condition (7) is satisfied for an eigenvector of $\mu_{1}$.

We next discuss graphs which have an eigenvector satisfying (7) with the eigenvalue $\mu=2 r_{1}>2$. Recall that by (14) $r_{1} \mid k$. By counting the edges connecting the vertices in two consecutive levels one can show (as for $\mu=2$ ) that the number of vertices satisfies

$$
|G|=2^{\left(k / r_{1}\right)} n_{0}
$$

Also, since any vertex $u_{1} \in G_{1}$ has $r_{1}$ distinct neighbors in $G_{0}$,

$$
n_{0} \geq r_{1} .
$$

It is easy to construct examples of regular graphs which have eigenvectors with the eigenvalue $\mu>2$ satisfying $(\overline{0})$; the construction is similar to that for $\mu=2$.

We summarize the previous results:

Theorem 13. Let $G$ be a k-regular graph which has an eigenvector of $\Delta$ with an eigenvalue $\mu$ satisfying (7). Then $G$ is bipartite, $\mu=2 l$ is an even integer dividing $2 k$, the number of vertices of $G$ is divisible by $2^{(k / l)}$, and for $n_{0} \geq l$ there exist such graphs with $n=2^{(k / l)} n_{0}$ vertices.

\section{REFERENCES}

[Big93] N. Biggs. Algebraic graph theory (2nd ed). Cambridge Univ. Press, 1993.

[Bol98] B. Bollobás. Modern Graph Theory, Springer Verlag, New York, 1998.

[Bou] I. Bouwer. On edge but not vertex transitive regular graphs. J. Comb. Th. B, 12:3240, 1972.

[Fie] M. Fiedler. Some minimax problems for graphs. Discr. Math, 121:65-74, 1993.

[Fos] R. Foster. The average impedance of an electrical network. Contib. to Applied. Mechanics (Reissner Ann. Volume), Edwards Bros, 333-340, 1949. 
[God81] C. Godsil. Equiarboreal graphs. Combinatorica, 1:163-167, 1981.

[Kir] F. Kirchhoff. Über die Auflösung der Gleichungen, auf welche man bei der Untersuchung der linearen Verteilung galvanischer Ströme gefürt wird. Ann. Phys. Chem. 72:497-508, 1847.

[Nil] A. Nilli. On the second eigenvalue of a graph. Discr. Math, 91:207-210, 1991.

[OPS] B. Osgood, R. Phillips and P. Sarnak. Extremals of Determinants of Laplacians. J. Func. Anal, 80:148-211, 1988.

[Riv99] I. Rivin. Growth in free groups (and other stories). xxx.lanl.gov preprint math.CO/9911076.

[Sch] A. Schrijver. Theory of linear and integer programming. John Wiley \& Sons, 1990.

[Val] L. Valdes. Extremal properties of spanning trees in cubic graphs. Congr. Numer, 85:143-160, 1991.

First Author: Mathematics Department, University of Chicago., Second author: Mathematics Department, University of Manchester and Mathematics Department, Temple University

E-mail address: rivin@ma.man.ac.uk, jakobson@math.uchicago.edu 